\title{
Mining meiosis with genomic models
}

Explaining the genetic basis of the processes of homologous chromosome pairing, bivalent formation, recombination and faithful segregation during meiosis I (MI) has been a vexing problem in biology. Gerton and Hawley ${ }^{1}$ have reviewed the exciting perspectives on these processes, which have been extracted mostly from the 'who-where-when' type of forward-genetics explorations in the widely known pairingrecombination-segregation (PRS) paradigm that is seen in model organisms. The general consensus that has emerged from these studies is that in spite of the significant differences in the structure of key components such as the synaptonemal complex and kinetochore in evolutionarily distant model organisms Saccharomyces cereviseae, Caenorhabditis elegans, Drosophila melanogaster and Arabidopsis thaliana their meiotic functions are highly conserved. In silico searches for functional meiotic proteins show that these organelles are made up of functional analogues. ${ }^{2}$. Bioinformatics and gene profiling have identified more than 300 genes that are implicated in meiosis ${ }^{3,4}$. This information pool needs to be validated and further enhanced by reverse-genetics approaches using genomic models.

Genetic domestication and mutation analysis have been instrumental in selecting classical genetic model organisms. By contrast, the choice of a genomic model is dictated by its unique features that can illuminate basic biology, rather than prior genetic domestication. Most of the models used so far have allowed only partial insight into a given biological phenomenon. The use of various non-classical models could correct the situation ${ }^{6}$. We argue that the dipteran insect Sciara coprophila, or fungal gnat, could provide valuable insight into the biology ofmeiosis.

Sciarids have avoided the highly conserved PRS pathway that is seen in bivalent-forming organisms (reviewed in REF. 7) and opted for a unique means to achieve haploidy during male meiosis. In the absence of pairing and recombination during MI, there is no apparent need for the proteins that regulate processes (REF 8 and the references therein) such as the formation of a synaptonemal complex, the formation and resolution of chiasmata and the recruitment of proteins such as monopolin for sister kinetochore coorientation'. There are other unique features in the male meiosis of sciarids. Instead of a bipolar spindle seen during bivalent MI, a monopolar halfspindle is formed.

Moreover, meiosis II (MII) is unique in these insects because only one bipolar spindle is formed instead of the two bipolar spindles that are seen during bivalent MII. It is generally believed that spindle formation can occur even in the absence of chromosomes ${ }^{10}$. An MII with a single bipolar spindle provides an exception to this dogma, because the second spindle is not formed in the absence of the paternal set of chromosomes.

Sciarid males undergo a programmed genome elimination(PGE) in both MI and MII (REFS 11,12). The centromere-kinetochore complex of the maternal autosomes works like a molecular switch - the complex is inactive and is not attached to the microtubules during MI, but is activated during MII when it attaches to the microtubules to undergo chromatid segregation on a bipolar spindle, thereby defying the search-capturestabilize type of microtubule-kinetochore interactions that are normally seen in bivalent meioses. The maternal X chromosome continues to have an inactive kinetochore during MII and does not attach to any microtubules. Instead, it does not participate in the chromatid segregation on the MII bipolar spindle and lies precociously near one pole, and is therefore passively transferred to one of the daughter cells. The kinetochores of both the maternal and paternal chromosome sets are ultrastructurally similar ${ }^{13}$, which suggests the involvement of unique molecular mechanisms in the programmed and selective activationinactivation of the kinetochores in space and time. A significant biological consequence of both PGE and the directed non-disjuncton of the maternal X sister chromatids during MII is the production of an unusual $n+1$ sperm.

Cohesin holds the sister chromatids of both the eliminated paternal set and the surviving maternal set of chromosomes, thereby adding a new dimension to its already known crucial roles. There seems to be no requirement for the meiosis-specific cohesin complex (formed by the replacement of the Scc1/Rad21 subunit of the mitotic cohesin complex with the meiosis-specific Rec8 cohesin subunit) that is required for a two-step resolution of sister chromatid cohesion during the PRS pathway (REFS 14,15 and the references therein). Unlike the dissolution of cohesion between the sister chromatids of the maternal autosomes, which are aligned on a bipolar spindle during MII, the dissolution of cohesion between the sister chromatids of the maternal X chromosome, which are precociously placed distant from the maternal autosomes near one of the poles, is poorly understood. Examining the protein networks that exist in such an altered proteomic environment should explain the basic biology of meiotic celtycle organization and function.

Selective chromosome elimination also occurs during early embryonic (mitotic) divisions in sciarids, adding a new dimension to the genome elimination that is seen during male meiosis. The signals involved in 
selectively targeting a particular chromosome for elimination in the early embryonic mitoses, and quenching these signals to ensure that the same genomic constitution exists in all the cells, are unique to sciarids. The process is linked to a unique type of sex determination, which depends on the number of X chromosomes that are eliminated during these early embryonic divisions ${ }^{16}$ and is implicated in a unique chromosomal form of imprinting ${ }^{17}$.

PGE also has biological consequences that are similar to the elimination of three of the four genomes as polar bodies that occurs during female meiosis in mammals. In the sciarid male meiosis, three naked genomes are eliminated, which prevents the formation of three sperm cells. Nevertheless, the developmental output in both female mammals and male sciarid remains same - the elimination of three genomes and formation of a single haploid sex cell.

All our cherished ideas of meiosis, as understood from the classical models, are bound to be put to the test as we learn from $S$. coprophila and other non-classical genomic models.

\section{R. M. Ranganath* \& G. Venkatachalaiah}

${ }^{*}$ Cytogenetics and Developmental Biology Laboratory, Department of Botany and Cytogenetics Laboratory, Department of Zoology, Bangalore University, Bangalore 560056, India.

1. Gerton, J. L. \& Hawley, R. S. Homologous chromosome interactions in meiosis: diversity amidst conservation. Nature Rev. Genet. 6, 477-487 (2005).

2. Bogdanov, Y. F., Dadashev, S. Y. \& Grishaeva, T. M. In Silico search for the functionally similar proteins involved in meiosis and recombination in evolutionarily distant organisms.

In Silico Biol. 3, 173-185 (2003).

3. Barchi, M. \& Jasin, M. Seeking new meiotic genes. Proc. Natl Acad. Sci. USA 100, 1528715289 (2003).

4. Critchlow, H. M., Payne, A. \& Griffin, D. K. Genes and proteins involved in meiosis. Cytogenet. Genome Res. 105, 4-10 (2004).

5. Davis, R. H. The age of model organisms. Nature Rev. Genet. 5, 69-76 (2004).

6. Golstein, P., Aubry, L. \& Levraud, J. -P. Cell-death alternative model organisms: which and why? Nature Rev. Mol. Cell Biol. 4, 798-807 (2003).

7. Marston, A. L. \& Amon, A. Meiosis: cell cycle controls shuffle and deal. Nature Rev. Mol. Cell Biol. 5, 983-997 (2004).

8. Barr, F. A., Sillje, H. H. W. \& Nigg, E. A. Polo -like kinases and the orchestration of cell division. Nature Rev. Mol. Cell Biol. 5, 429-441 (2004).

9. Toth, A. et al. Functional genomics identifies monopolin: a kinetochore protein required for segregation of ho mologues during meiosis I. Cell 103, 1155-1168 (2000).

10. Hyams, J. Look Ma! No chromosomes. Nature 382, 397-398 (1997).

11. Esteban, M. R., Campos, M. C., Perondini, A. L. \& Goday, C. Role of microtubule and microtubule organizing centers on meiotic chromosome elimination in Sciara oscellaris. J. Cell Sci. 110, 721-730 (1997).

12. Goday, C. \& Esteban, M. R. Chromosome elimination in sciarid flies. Bioessays 23, 242-250 (2001).

13. Kubai, D. F. Meiosis in Sciara coprophila: structure of the spindle and ch romosome behaviour during the first meiotic division. J. Cell Biol. 93, 655-669 (1982).

14. Kitajima, T. S., Yokobayashi, S., Yamamoto, M. \& Watanabe, Y.

Distinct cohesin complexes organize meiotic chromosome domains. Science $\mathbf{3 0 0}$, 1152-1155 (2003).

15. Kitajima, T. S., Kawashima, S. A. \& Watanabe, Y. The conserved kinetochore protein shugoshin protects centromere cohesion during meiosis. Nature 427, 510-517 (2004).

16. Endow, S. A. Programmed to stay together. Nature 384, 412-413 (1996).

17. Crouse, H. V., Brown, A. \& Mumford, B. C. L. L chromosome inheritance and the problem of chromosome imprinting in Sciara (Sciaridae, Diptera). Chromosoma 34, 324-339 (1971). 